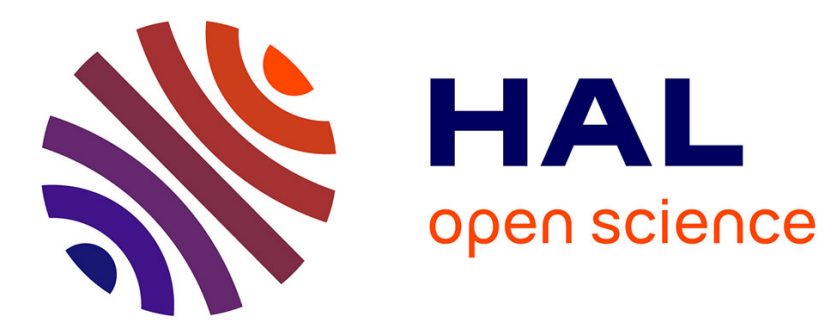

\title{
Inferring Equation-Based Models from Agent-Based Models : A Case Study in Competition Dynamics
}

Doanh Nguyen Ngoc, Patrick Taillandier, Alexis Drogoul, Pierre Auger

\section{To cite this version:}

Doanh Nguyen Ngoc, Patrick Taillandier, Alexis Drogoul, Pierre Auger. Inferring Equation-Based Models from Agent-Based Models: A Case Study in Competition Dynamics. Principles and Practice of Multi-Agent Systems, 2010, Kolkata, India. pp.413-427, 10.1007/978-3-642-25920-3_30 . hal00689108

\section{HAL Id: hal-00689108 https://hal.science/hal-00689108}

Submitted on 19 Apr 2012

HAL is a multi-disciplinary open access archive for the deposit and dissemination of scientific research documents, whether they are published or not. The documents may come from teaching and research institutions in France or abroad, or from public or private research centers.
L'archive ouverte pluridisciplinaire HAL, est destinée au dépôt et à la diffusion de documents scientifiques de niveau recherche, publiés ou non, émanant des établissements d'enseignement et de recherche français ou étrangers, des laboratoires publics ou privés. 


\title{
Inferring Equation-Based Models from Agent-Based Models: A Case Study in Competition Dynamics
}

\author{
Nguyen Ngoc Doanh ${ }^{1,2}$, Patrick Taillandier ${ }^{1,2}$, Alexis Drogoul ${ }^{1,2}$, Pierre Auger ${ }^{2,3}$ \\ ${ }^{1} \mathrm{MSI}$, IFI, 42 Ta Quang Buu street, Hai Ba Trung District, Hanoi, Vietnam. \\ ${ }^{2}$ UMMISCO, UMI 209, IRD/UPMC, 32 Henri Varagnat, 93143 Bondy Cedex, France. \\ ${ }^{3}$ LMPD, University Cadi Ayyad, Marrakech, Morocco. \\ Emails: doanhbondy@gmail.com, patrick.taillandier@gmail.com, \\ alexis.drogoul@gmail.com, pierre.auger@ird.fr .
}

\begin{abstract}
Two types of model, equation-based models (EBMs) and agentbased models (ABMs) are now widely used in modeling ecological complex systems and seem not to be reconciled. While ABMs can help in exploring and explaining the local causes of global phenomena, EBMs are useful for predicting their long-term evolution without having to explore them through simulated experiments. In this paper, we show that it is possible to use an $\mathrm{ABM}$ to infer an EBM. Base on the case study, a dynamics of two competing species, we illustrate our methodology through the presentation of two models: an ABM and an EBM. We also show that the two models give the same results on coexistence of the two competing species.
\end{abstract}

\section{Categories and Subject Descriptors}

I.6.0 [Simulation and Modeling]: General

\section{General Terms}

Design, Theory.

\section{Keywords}

Agent-Based Models, Equation-Based Models, Population Dynamics, Complex Systems.

\section{INTRODUCTION}

In modeling ecological complex systems, two widely accepted models coexist: agent-based models (ABMs) and equation-based models (EBMs). Each of these two models has its own strengths and weakness ([6], [12]) depending on the purpose of study. EBMs, on one hand, play as compartment models and operate on global laws generally, defined by the equations that apply to all members of the compartments. For example, in early ecological models, the state variables (compartments) in the models of population dynamics was often chosen as the total population densities and the model was a set of nonlinear, coupled, ordinary differential equations or discrete equations [8]. In such classical models, the agents (individuals) are assumed to be homogenous and well mixed: they are all treated as identical. The benefit of these simple EBMs is that they can be handled analytically. However, given this previous assumption, they cannot be very realistic. In recent years, some "more realistic" EBMs have been developed, which are aimed at taking different categories of agents into account. These EBMs involve a large number of variables and are in general difficult to handle analytically. However, in most cases, it is possible to consider different time scales: a fast one for processes operating at the agent level; and a slow one at the levels of the population and the community. It is then possible to use the "variable aggregation" method, deriving a reduced model which governs a few global variables at the slow time scale while taking into account all the processes going on at the agent level [9]. One can also find in [9] some interesting examples of EBMs in which different categories of agents were considered, and for which aggregation methods were used successfully to proceed analysis of the EBMs.

ABMs, on the other hand, are natural representations of real ecological systems [10]. The obvious reason for using ABMs to model a real ecological system is that agents are building blocks of ecological systems. The properties and behaviors of agents determine the properties of the systems that the agents compose. In ecological systems, agents are not identical and do not stay the same all their life: all that an agent does-grow, develop, acquire resources, reproduce, interact - depends on its internal and external environments and it modifies both with its actions. ABMs are then particularly adapted to represent and understand the emergence of global dynamics among heterogeneous agents sharing common environmental constraints. In comparison to EBMs, ABMs are much more realistic with respect to the data available in the field and thus are easier to test in scientific process. But being closer to reality usually means having to define, calibrate and determine much more parameter values than in EBMs and analysis problems then arise.

To summarize, both ABMs and EBMs address, with its own point of view, the same problems in ecological complex systems. Each of these modeling approaches answers different, yet 
complementary, questions. While ABMs can help in exploring and explaining the local causes of global phenomena, EBMs are useful for predicting their long-term evolution without having to explore them through simulated experiments.

Therefore, it is primary to couple these two modeling approaches when studying ecological complex systems. There are many alternative ways for coupling these two approaches. For instance, the process can be completely top-down: by distributing global parameters of a given EBM to obtain local parameters of a related ABM. We refer to our previous contribution [12] for this way. It can also be bottom-up, by extracting local parameters of a given ABM to obtain global parameters of inferred EBM. Several studies seem to be related to this way can be found in ([15], [16]). In these contributions, the authors tried to abstract ABMs by introducing mathematical formalism [15] as well as moment approximations of ABMs [16].

In this paper, the novel issue we explore is closed to the "bottomup" way. Based on the case study, a dynamics of two competing species, we represent how to infer an EBM from a given ABM. Unlike in the previous studies ([15], [16]), we extract local parameters to obtain global ones. For instance, we show that an EBM can be built from a given ABM by considering $A B M$ as a virtual laboratory to test and to see effects of parameters. Once we observe the effects of parameters, we choose parameters to build EBMs. We also show that the two models give the same results on coexistence of the two competing species.

The paper is organized as follows. In section 2, we present the case study -the dynamics of two competing species. The ABM implemented for the case study is presented in section 3 . We then present in section 4 how we extract global parameters in order to build an EBM. We present the EBM and its result in section 5, we also comparison result of the inferred EBM and the ABM. Section 6 is dedicated for conclusion and perspectives.

\section{CASE STUDY: COMPETITION DYNAMICS}

We consider a system of two species competing for a common resource. We are interested in pre-emptive competition, i.e., one of the two species will end up extinction if it has smaller number of individuals at the beginning. There are a lot of such complex systems in the reality where two species coexist. The aim is to figure out under which conditions two species coexist in the preemption dynamics.

In the next sections, we shall present two models which can explain the coexistence of two species in pre-emption dynamics. To do that, we first present an ABM for dynamics of two competing species. We then test the $\mathrm{ABM}$ to figure out parameters and conditions under which two species coexist. We based on these results to build an EBM which has provable result on coexistence of two competing species.

\section{AGENT-BASED MODEL}

In this section, we propose a description following the ODD protocol [1] of our ABM.

\subsection{Overview}

\subsubsection{Purpose}

The purpose of the model is to study the dynamic of two species when these ones are in competition and when the total quantity of food existing in the environment is stable.

\subsubsection{State variables and scales}

In our model, each individual of each species is represented as an "agent" that is located in a continuous environment. The food patches are also modeled as agents.

\subsubsection{Environment}

We chose to model the environment as a 2D plane of a specific size $(300 \times 300)$. The agents can not go out this environment.

\subsubsection{Food Patch Agent (FPA)}

Each food patch is represented by an agent (FPA). A FPA has for geometry a circle of which the area depends of the quantity of food contained in the food patch. The attributes of FPAs are described in Table 1.

\subsubsection{Species Individual Agent (SIA)}

Each species individual is represented by an agent (SIA) that has a point for geometry. The attributes of these agents are described in Table 2.

Table 1. Attributes of the food patch agents

\begin{tabular}{|l|l|l|}
\hline Attribute name & Brief description & Value \\
\hline Location & $\begin{array}{l}\text { X and Y coordinates of the food patch } \\
\text { (its center) }\end{array}$ & Random \\
\hline $\begin{array}{l}\text { Food } \\
\text { Production }\end{array}$ & $\begin{array}{l}\text { quantity of food produced at each } \\
\text { simulation step (in the food patch) }\end{array}$ & 30 \\
\hline Food & $\begin{array}{l}\text { current quantity of food contained in } \\
\text { the food patch (when null, the agent } \\
\text { dies) }\end{array}$ & - \\
\hline Max food & $\begin{array}{l}\text { maximal quantity of food in a food } \\
\text { patch }\end{array}$ & 100 \\
\hline $\begin{array}{l}\text { FoodArea } \\
\text { coefficient }\end{array}$ & $\begin{array}{l}\text { coefficient that links the food quantity } \\
\text { to the food patch area }\end{array}$ & 5 \\
\hline Area & area of the food patch & - \\
\hline
\end{tabular}

\subsubsection{Process Overview and Scheduling}

At each simulation step, the SIAs act, then the FPAs evolve.

The evolution of the FPAs is very simple (Figure 1): if there is no more food in the food patch, the agent dies, otherwise, the quantity of food evolves according to the food production attribute. Then the new area of the food patch is computed. When a FPA dies, a new one is created (at a random location). This 
mechanism allows to keep a stable quantity of food in the environment.

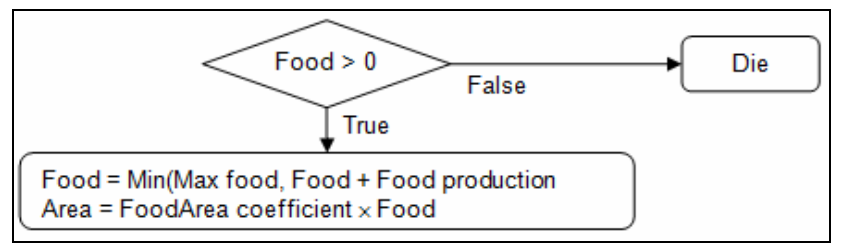

Figure 1. Food patch agent evolution (at each simulation step)

Concerning the SIAs, their general behavior consists in trying to survive (Figure 2). They have the capacity to move, to eat and to reproduce. They gain energy by eating food (in a food patch) and lose it, when fighting on a patch with member of the other species. When a SIA finds a food patch, it remains in this one as long as the proportion of agents of the same species is high enough. The choice of a new food patch is done randomly between the food patch perceived by the agent.

Table 2. Attributes of the species individual agents

\begin{tabular}{|l|l|l|}
\hline Attribute name & Brief description & Value \\
\hline Location & X and Y coordinates of the agent & - \\
\hline $\begin{array}{l}\text { Displacement } \\
\text { range }\end{array}$ & $\begin{array}{l}\text { maximal distance of displacement per } \\
\text { step }\end{array}$ & 100 \\
\hline Energy & $\begin{array}{l}\text { quantity of energy (when null, the } \\
\text { agent dies) }\end{array}$ & - \\
\hline Max energy & maximum quantity of energy & 30 \\
\hline $\begin{array}{l}\text { Energy } \\
\text { consumption }\end{array}$ & $\begin{array}{l}\text { quantity of energy consumed at each } \\
\text { simulation step }\end{array}$ & 2 \\
\hline $\begin{array}{l}\text { Extra } \\
\text { competition } \\
\text { coefficient }\end{array}$ & $\begin{array}{l}\text { coefficient of energy lost per } \\
\text { simulation step due to competition } \\
\text { with individuals of the other species }\end{array}$ & 10 \\
\hline $\begin{array}{l}\text { Energy } \\
\text { reproduction }\end{array}$ & $\begin{array}{l}\text { energy lost during the reproduction } \\
\text { (the energy of the offspring will be } \\
\text { equal to the energy reproduction / } \\
\text { number of offspring) }\end{array}$ & 10 \\
\hline $\begin{array}{l}\text { Reproduction } \\
\text { probability }\end{array}$ & $\begin{array}{l}\text { probability that an individual } \\
\text { reproduces at each simulation step }\end{array}$ & 0.01 \\
\hline $\begin{array}{l}\text { Reproduction } \\
\text { Time }\end{array}$ & $\begin{array}{l}\text { minimal number of steps between two } \\
\text { reproductions }\end{array}$ & 20 \\
\hline Max offspring & $\begin{array}{l}\text { maximal number of offspring that an } \\
\text { individual can have when reproducing }\end{array}$ & 3 \\
\hline $\begin{array}{l}\text { Max } \\
\text { consumption }\end{array}$ & $\begin{array}{l}\text { maximal quantity of food that an } \\
\text { individual agent can eat at each } \\
\text { simulation step }\end{array}$ & 4 \\
\hline $\begin{array}{l}\text { Tolerance } \\
\text { other species }\end{array}$ & $\begin{array}{l}\text { proportion of individual agents of } \\
\text { other species from which the agent is } \\
\text { going to leave a food patch. Defined } \\
\text { the strategy of the agent }\end{array}$ & 0.3 \\
\hline
\end{tabular}

\subsection{Design concepts}

\subsubsection{Emergence}

In this model, the emergent aspect concerns the population dynamics resulting from the interaction between the two species of SIAs and the FPAs; in particular the creation of groups of individual of the same species in a same food patch. It is influenced by different parameters: the extra competition coefficient, the tolerance for others, the food production, etc.

\subsubsection{Fitness}

Each individual does not have an explicit fitness function to optimise. However, the implicit fitness of a species concerns its survival. Thus the more agents of this species, the higher the fitness for this species will be.

\subsubsection{Adaptation}

The adaptive trait of the SIAs comes from their capacity to not tolerate a high proportion of agent of other species. This property allows them to avoid food patches with too much competition.

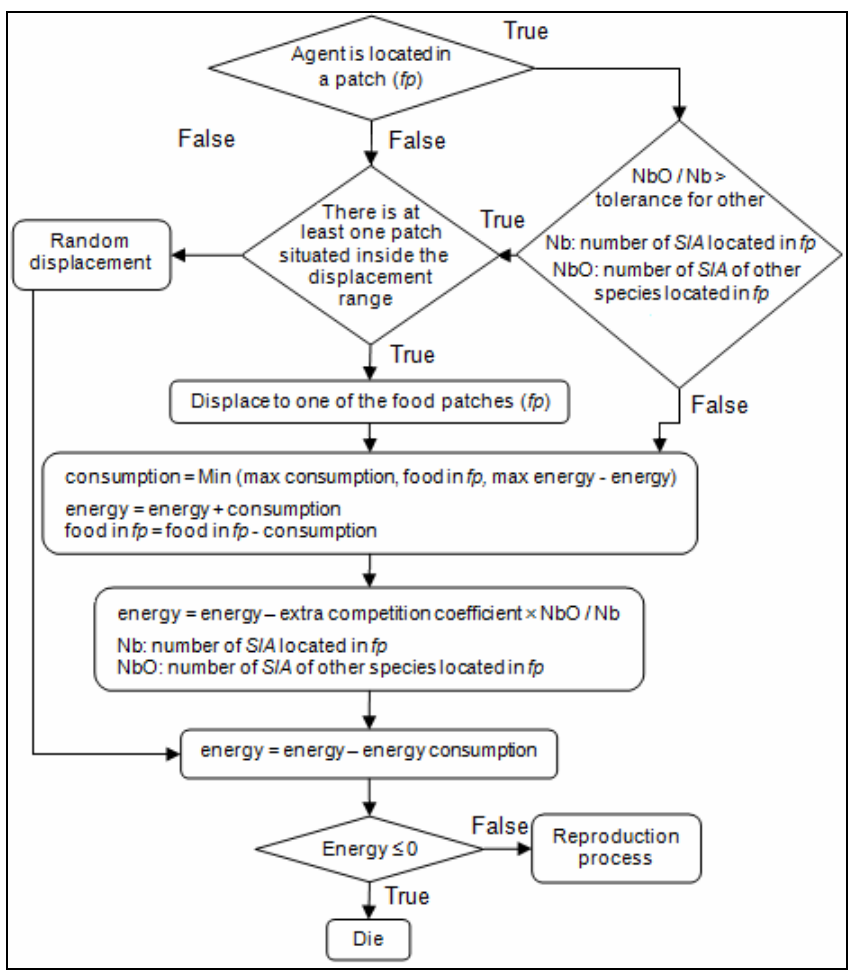

Figure 2. Species individual agent behaviour (at each simulation step)

\subsubsection{Interaction}

There are two kinds of interaction between agents: the interaction between the SIAs and the FPAs (the SIAs eat food contains in FPA) and the interaction between SIAs of different species (competition and non tolerance to agents of other species).

\subsubsection{Sensing}

The SIAs know the number of agents (of the same species and of other species) that share the same food patch as them. They can 
detect food patches which geometry overlaps their seeing range (displacement range).

\subsubsection{Stochasticity}

The stochasticity is involved in the repartition of the food patches in the environment. It is also involved in the initial food contained in the food patches. At last, it is involved in the choice of a food patch by the SIA: these ones randomly choose a food patch among the perceived ones.

\subsubsection{Collectives}

SIAs of the same species sharing a same food patch formed an implicit group. Higher the number of agents in this group, the stronger will be its defense against SIAs from other species, but more quickly the food contained in the food patch will be consumed.

\subsubsection{Observation}

Various observations are available in this model from an omniscient perspective. However, as we study the population dynamics, a first observation is the evolution of the number of agents of the two species. The repartition of the species in the different food patches is as well an interesting observation.

\subsection{Details}

\subsubsection{Initialisation}

At the initialisation, the FPAs and the SIAs are randomly placed in the environment. The quantity of food in each food patch is randomly drawn between [1, max food]. In the way the quantity of energy of each SIA is randomly drawn between [1, max energy]. The initial number of SIAs of each species is 50 . The number of FPAs (which is constant) is equals to 10 .

\subsubsection{Simulation implementation}

Since few years, many simulation platforms dedicated to the implementation of agent-based models have been developed. We can cite as examples GAMA [2], Mason [3], Repast [4], NetLogo [5]. In this work, we chose to develop our simulation with the GAMA platform. This platform provides a complete modelling and simulation environment for building spatially explicit multiagent simulations. In particular, it integrates powerful spatial analysis tools coming from Geographic Information Systems (GIS) allowing to give agents a geometry and spatial analysis capacities.

\subsubsection{Test the model}

Figure 3 shows examples of results concerning the distribution of individuals at several simulation steps. This figure shows that at step 1 , the individuals are randomly located in the food patches; then at step 3, groups of individuals of the same species are beginning to form; at last, at step 20, these groups are uniform (only composed of individuals of the same species).

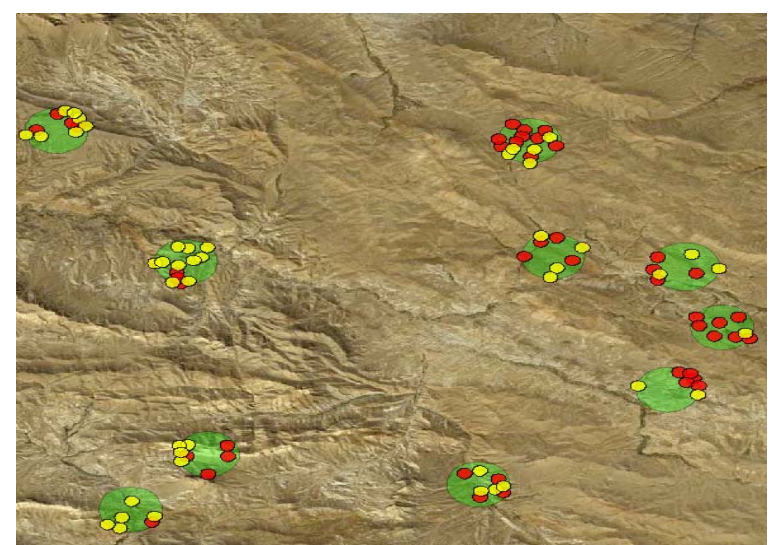

a)

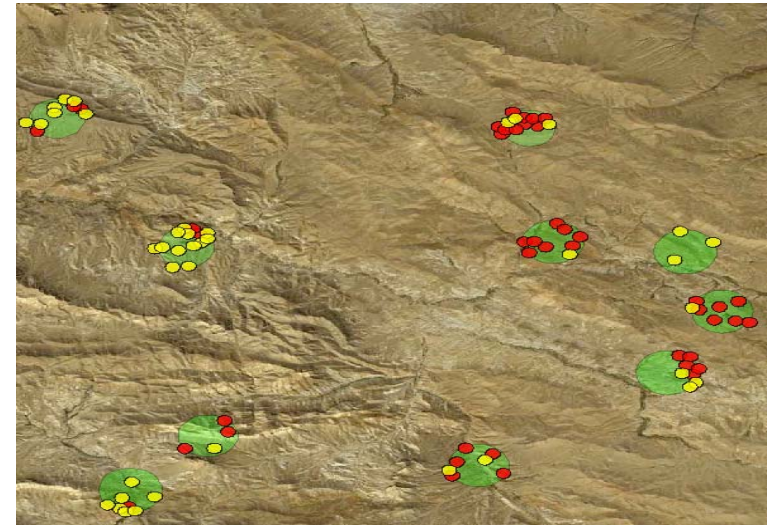

b)

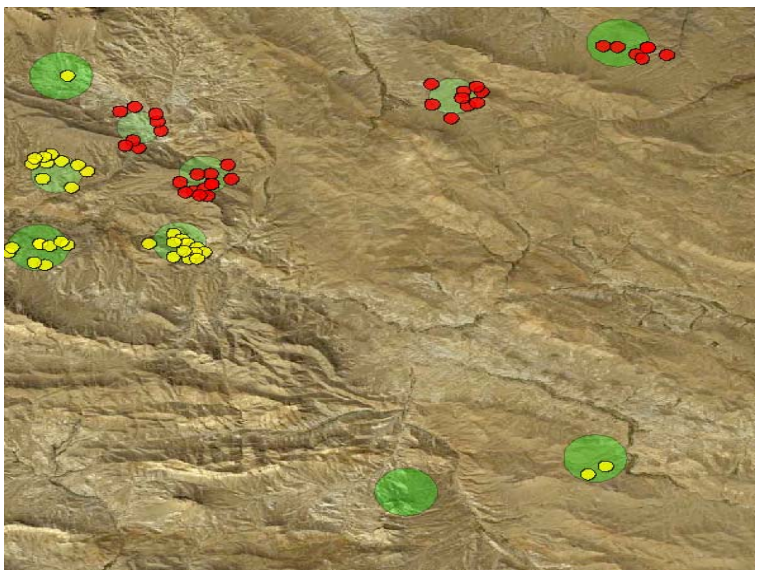

C)

Figure 3. Distribution of individuals in several simulation steps. In red, Species $A$, in yellow Species $B$. a) at step 1 b) at step 3, c) at step 20

Figure 4 gives the simulation results obtained for the population evolution during the first 20 steps. In order to build this graphic, we carried out 30 simulations and we computed the means. The goal was to limit the stochastic bias of the model. First, one can observe that the two populations similarly evolve. The population is stable during the first three steps, and then it decreases until reaching an equilibrium point. 


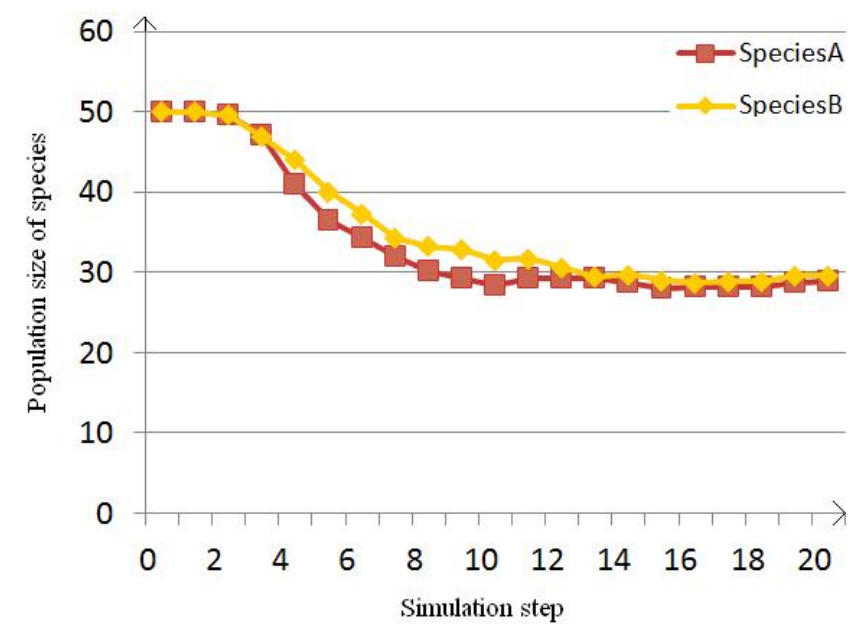

Figure 4. Evolution of the number of individuals of each species (mean of 30 simulations).

\subsubsection{Remarks}

We pay our attention on the key result concerning to the parameter "tolerance for other species". Actually, this parameter, which defines the strategy used by the SIs to survive, has a deep impact on the generation of SIs aggressive/defensive groups. When the value of this parameter is high enough we can observe the formation of groups composed of SIs of the same species. These groups can allow to the IC to survive by combining their strength. In this context, we test two values for "tolerance for other species" parameters of the IC individual: 0 and 0.3 . Figure |ref\{ch4fig45\} shows examples of simulation results obtained with these two parameter values: figure $\mid r e f\{c h 4 f i g 45\}$ a) corresponding to the case where the IC individuals do not have a specific strategy: they randomly choose a food patch and then do not move until this patch does not contain food anymore. One can see that IC will go extinct. In figure $\mid \operatorname{ref}\{\operatorname{ch} 4 f i g 45\}$ b), the IC individuals try to avoid the SC individuals by searching food patches where there are not too many SC individuals. With this second parameter value, the IC individuals tend to form big groups that "invade" patches without SC individuals. These tactics of IC individuals lead to distribution of IC individuals on patches. To the first tactics which corresponds to the case where parameter "tolerance for other species" of the IC individual equals to 0 , IC individuals distribute randomly on food patches. To the second tactics which corresponds to the case where parameter "tolerance for other species" of the IC individual equals to 0.3 , IC individuals are likely to distribute on food patches where there are few SC individuals.

\section{METHODOLOGY}

EBMs are compartment models which operate on global laws generally, defined by the equations that apply to all members of the compartments. In such models, the individuals are assumed to be homogenous and well mixed: they are all treated as identical. Moreover, EBMs do not have "real" environment which allows individuals eat, move, reproduce and interact with others individuals. Therefore, our methodology basically is to abstract/simplify local parameters and to translate them into equations of compartments.

\subsection{First step: simplification and abstraction environment and translate it into equations.}

The first step of our methodology is to simplify the environment, i.e. to get rid of many local properties of the environment such as its topology, its dynamics, its nature and so on. Several questions are raised in this step:

- the homogeneity of the environment

- its discretization, i.e. patchy environment

- the perception it can offer to species (for instance, are there any refuge for species?) and so on

\subsection{Second step: analysis and abstract local behaviors of agents and translate them into equations}

The second step is to analyze, one by one, the local behaviors of the agents. We then abstract and translate them into global parameters in equations. Each of these "abstractions" is tested and validated with respect to the results obtained with the simulations of the IBM.

Each step consists in:

- Building several models related to the global parameters we choose

- Exploring their dynamics and "validate" each of them with respect to the IBM simulation results

- Choosing the most relevant value of the parameters

\section{INSTANTIATION ON THE CASE STUDY}

In this section, we show how global parameters can be extracted from the local parameters of the IBM. An EBM consists of compartments which are usually chosen as population densities in ecology systems. We, therefore, consider compartments in our EBM as population densities. Others factors and parameters have effects on these parameters. These effects, of course, are represented by parameters in an evolution function of the compartment-population densities. We now investigate, by using the methodology presented above, how to represent these factors and parameters and such an evolution function in our EBM.

\subsection{Simplification and abstraction environment and translate it into equations}

The first factor is environment. The environment in the IBM is very complex. It, however, should be simple in our EBM. According to the above remarks, we consider the environment in our EBM as a simplest case of patchy environment, i.e. two patches environment. This leads to the fact that there are four compartments of the two species in two patches: species 1 (species 2, respectively) on patch 1 , species 1 (species 2, respectively) on patch 2 . 


\subsection{Analysis and abstraction local behaviors of individuals and translate them into equations}

\subsubsection{Reproduction probability}

This factor represents the probability that an individual reproduces after a certain number of simulation steps. It means that the population densities increase when values of this factor increases. The corresponding global parameter is growth rate which has positive effect on evolution of population densities.

\subsubsection{Competition parameters}

In the IBM, there are two kinds of competition: the intracompetition which represents the competition among individuals of the same species, and the extra-competition which represents the competition among individuals of different species. To represent this factor in EBM, the classic Lotka-Volterra competition model \cite $\{$ M89\} seems to be a good candidate. We note that, in the IBM,, individuals compete with others for food. The competition, therefore, takes place on the patches.

Following this analysis, we propose therefore to use the classic Lotka-Volterra competition model to represent the evolution of population densities on both patches.

\subsubsection{Movement/migration}

Another important factor is the movement of individuals. In the IBM, SC individuals move randomly in the environment to search for food patches. It is, therefore, assumed that SC always stay on some food patches. IC individuals have two kinds of movement: the first one is a random move and the second one is a SC density dependent move, i.e. the IC individuals are more likely to move to patches where there are few SC individuals. These tactics of movement, on the fast time scale, lead to the distribution of IC on the patches (\cite\{ABPSN08a\}, \cite\{ABPSS08b\}, appendix (ref\{appendix0\}). Being in mind that we do not have a real environment in our EBM. Therefore, we represent the number of immigrations and emigrations on each patch by using a function of density, i.e. a migration function. This function must increase when the other species population density increase. To simplify, we choose a homogenous linear function of population density, i.e. a straight line.

\subsubsection{Time scales}

The important point is that in the IBM, all the agent behaviors are not triggered every simulation step. Indeed, while the agents move at each step, they reproduce and interact only at specific simulation steps. Local dynamics (reproduction, interaction), therefore, seems to act on slow time scale than movement process. We represent the two time scale, in our EBM, by using small parameter $\varepsilon$ which is the ratio between two time scales.

In the next section, we use these global parameters to build our EBM. We also analysis the EBM and compare its results with the results of the ABM.

\section{EQUATION-BASED MODEL}

\subsection{Complete model}

We consider two competing species in two patch environment. Based on the above remarks, we further assume that two time scales are involved in the dynamics: a fast one corresponds to dispersal between patches and a slow for local population dynamics. According to these assumptions, the complete model reads as follows:

$$
\left\{\begin{array}{c}
\frac{d n_{11}}{d \tau}=\left(-\left(\alpha_{1} n_{21}\right) n_{11}+\left(\alpha_{2} n_{22}\right) n_{12}\right) \\
+\varepsilon r_{11} n_{11}\left(1-\frac{n_{11}}{K_{11}}-a_{121} \frac{n_{21}}{K_{11}}\right) \\
\frac{d n_{12}}{d \tau}=\left(\left(\alpha_{1} n_{21}\right) n_{11}-\left(\alpha_{2} n_{22}\right) n_{12}\right) \\
+\varepsilon r_{12} n_{12}\left(1-\frac{n_{12}}{K_{12}}-a_{122} \frac{n_{22}}{K_{12}}\right) \\
+\varepsilon r_{21} n_{21}\left(1-\frac{n_{21}}{K_{21}}-a_{211} \frac{n_{11}}{K_{21}}\right) \\
\frac{d n_{21}}{d \tau}=\left(-\left(\beta_{1} n_{11}\right) n_{21}+\left(\beta_{2} n_{12}\right) n_{22}\right) \\
\quad+\varepsilon r_{22} n_{22}\left(1-\frac{n_{22}}{K_{22}}-a_{212} \frac{n_{12}}{K_{22}}\right)
\end{array}\right.
$$

where $n_{1 j}$ is the density of species $A$ living on patch $j, n_{2 j}$ is the density of species $B$ living on patch $j j=1,2 . r r^{\prime} s$ and $K$ 's represent the growth rates and carrying capacities of species. Parameters $a_{12 j}$ and $a_{21 j}$ represent the competition coefficients showing the negative effect of species $A$ on species $B$ and species $B$ on species $A$ on patch $j, j=1,2$, respectively. $\alpha_{j}$ is the dispersal rate of the species $A$ leaving patch $j, j=1,2$, and $\beta_{j}$ is the dispersal rate of the species $B$ leaving patch $j, j=1,2$. $\varepsilon$ is the ratio between two time scales. The term with $\varepsilon$ corresponds to the slow time scale-birth death and competition processes; and the term without $\varepsilon$ corresponds to the fast time scale-migration process. In this model, individuals of a given species use the other species density-dependent migration in the sense that if there are many individuals of a given species on a given patch then individuals of the other species are more likely to leave that patch rapidly. We note that we are interested in preemptive competition locally on each patch and the conditions ensure for this case are given by

$$
a_{12 j} K_{2 j} / K_{1 j}>1, a_{21 j} K_{1 j} / K_{2 j}>1, \quad j=1,2 .
$$

We are going to use aggregation of variables methods in order to derive a reduced model [9]. The first step is to look for the existence of a stable and fast equilibrium. 


\subsection{Fast Equilibrium}

Fast equilibrium is the solution of the following system:

$$
\begin{gathered}
\alpha_{1} n_{11} n_{21}=\alpha_{2}\left(n_{1}-n_{11}\right)\left(n_{2}-n_{21}\right), \\
\beta_{1} n_{11} n_{21}=\beta_{2}\left(n_{1}-n_{11}\right)\left(n_{2}-n_{21}\right), \\
n_{1}=n_{11}+n_{12}, n_{2}=n_{21}+n_{22}
\end{gathered}
$$

It is easy to obtain that there are two stable and fast equilibria as follows:

$$
\begin{aligned}
& n_{11}{ }^{*}=n_{1}, n_{12}{ }^{*}=0 ; n_{21}{ }^{*}=0, n_{22}{ }^{*}=n_{2} \\
& \text { and } n_{11}{ }^{*}=0, n_{12}{ }^{*}=n_{1} ; n_{21}{ }^{*}=n_{2}, n_{22}{ }^{*}=0
\end{aligned}
$$

\subsection{Aggregated model}

Substitution of the fast equilibria into the complete model (1) leads to two reduced models as follows:

Model 1: is the model which corresponds to the equilibrium $n_{11}{ }^{*}=n_{1}, n_{12}{ }^{*}=0 ; n_{21}{ }^{*}=0, n_{22}{ }^{*}=n_{2}$.

$$
\left\{\begin{array}{l}
\frac{d n_{1}}{d t}=r_{11} n_{1}\left(1-\frac{n_{1}}{K_{11}}\right) \\
\frac{d n_{2}}{d t}=r_{22} n_{2}\left(1-\frac{n_{2}}{K_{22}}\right)
\end{array}\right.
$$

Model 2: is the model which corresponds to the equilibrium $n_{11}{ }^{*}=0, n_{12}{ }^{*}=n_{1} ; n_{21}{ }^{*}=n_{2}, n_{22}{ }^{*}=0$.

$$
\left\{\begin{array}{l}
\frac{d n_{1}}{d t}=r_{12} n_{1}\left(1-\frac{n_{1}}{K_{12}}\right) \\
\frac{d n_{2}}{d t}=r_{21} n_{2}\left(1-\frac{n_{2}}{K_{21}}\right)
\end{array}\right.
$$

The two aggregated models are two logistic models for two species on two patches. Model 1 corresponds to the case when all individuals of species $A$ are located on patch 1 while all individuals of species $B$ are located on patch 2 and Model 2 corresponds vice versa. This means that two species coexist globally in the patchy environment and each species has its own living patch. One can see that this result is exactly the same as the result in the ABM. Figure 5 shows the case of model 2 when all individuals of species $B$ are located on patch 1 while all individuals of species $A$ are located on patch 2 .

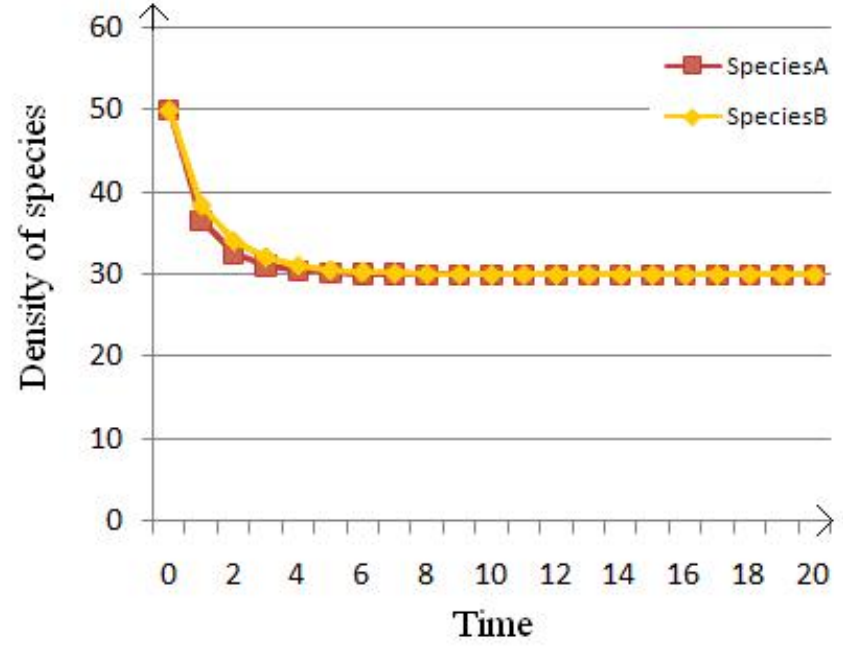

Figure 5. Two species coexist on two patches in model 2.

\section{CONCLUSION AND PERSPECTIVES}

In this paper, we proposed a methodology to infer an EBM from a given ABM. This methodology was illustrated through a case study concerning the competition of two species. Our idea is to consider as a virtual laboratory to test and to see effects of parameters and then to choose parameters to build the EBM. We also showed that the coexistence result of the obtained EBM is the same as that of the ABM. We conclude that these two techniques do not compete with each other: they instead tend to be ideally complementary with respect to the set of questions a modeler would want a model to answer. In this paper, we consider a simple case study of only two competing species. It would be interesting to consider more complex case studies of more than two competing species. It would be also interesting to couple these two types of model in modeling others ecological complex systems such as prey-predator systems, host-parasitoid systems and especially in epidemiology systems where it is impossible for one to test in order to get empirical observations and thus it is useful to use ABMs as a virtual laboratory. We would like to present these contributions in the near future.

\section{REFERENCES}

[1] Grimm, V., Berger, U., Bastiansen, F., Eliassen, S., Ginot, V., Giske, J., Goss-Custard, J., Grand, T. , Heinz, S.K., Huse, G., Huth, A., Jepsen, J.U., Jørgensen, C., Mooij, W.M., Müller, B., Pe'er, G., Piou, C., Railsback, S.F., Robbins, A.M., Robbins, M.M. , Rossmanith, E., Rüger, N., Strand, E., Souissi, S., Stillman, R.A., Vabo, R., Visser, U. and DeAngelis, D.L. 2006. A standard protocol for describing individual-based and agent-based models. Ecological Modelling 198 (1-2), pp. 115-126.

[2] Amouroux, E., Chu, T.Q, Boucher, A. and Drogoul, A. 2007. GAMA: an environment for implementing and running spatially explicit multi-agent simulations. International conference PRIMA, Bangkok, Thailand. 
[3] Luke, S., Cioffi-Revilla, C., Panait, L., Sullivan, K. and Balan, G. 2005. MASON: a multiagent simulation environment. Simulation, vol. 81, pp. 517-527.

[4] North, M.J., Collier, N.T. and Vos, J.R. 2006. Experiences Creating Three Implementations of the Repast Agent Modeling Toolkit. ACM Transactions on Modeling and Computer Simulation, v16(1), pp. 1-25.

[5] Tisue, S. and Wilensky. U. 2004. NetLogo: A Simple Environment for Modeling Complexity, ICCS.

[6] Fahse, L., Wissel, C., Grimm, V. 1998. Reconciling classical and individual-based aprroahes in theoretical population ecology : a protocol for extracting population parameters from individual-based models. American Naturalist 152 : 838 - 852 .

[7] Georiy, V. Bobashev, Goedecke, M.D., Yu, Jo F., Epstein, S. M. 2007. A hybrid epidemic model: Combining the advantages of agent-based and equation-based approaches. Winter Simulation Conference.

[8] Murray, J.D. 1989. Mathematical Biology, Springer Verlag.

[9] Auger, P., Bravo de la Parra, R., Poggiale, J.C., Sánchez, E., Nguyen Huu, T. 2008. Aggregation of variables and applications to population dynamics, In: P. Magal \& S. Ruan (Eds.), Structured Population Models in Biology and Epidemiology, Springer.

[10] Grimm, V. and Railsback, S.F. 2005. Individual-based Modeling and Ecology. Princeton University Press.
[11] Laubenbacher, R., Jarrah, A.S., Mortveit, H. and Ravi, S.S. 2007. A mathematical formalism for agent-based modeling, arXiv:08.01.0249v1 [cs. MA].

[12] Nguyen,N.D, Drogoul, A., Auger, P. 2008. Methodological Steps and Issues When Deriving Individual-Based Models from Equation-Based Models: A Case Study in Population Dynamics. PRIMA 2008, LNAI 5357, pp.295 -306.

[13] Eubank, S, Guclu, H., Anil Kumar, V. S., Marathe, A. Srinivasan, M. V., Toroczkai, Z. and Wang, N. 2004. Modelling disease outbreaks in realistic urban social networks. Nature 429, 184-190.

[14] Ohkusa, Y., and Sugawara, T. 2007. Application of an individual-based model with real data for transportation mode and location to pandemic influenza. Journal of Infection and Chemotherapy, Vol 13 (6), pp.380-389.

[15] Laubenbacher, R., Jarrah, A.S. , Mortveit, H., Ravi, S.S.: A mathematical formalism for agent-based modeling, arXiv:08.01.0249v1 [cs. MA], 2007.

[16] Law, R.,Dieckmann, Ulf.: Moment approximations of individual-based models, http://www.iiasa.ac.at/Admin/PUB/Documents/IR-99043.pdf , 1999. 